\title{
Vitamin D shuts down T cell-mediated inflammation
}

\section{6 \\ complement \\ stimulates \\ T cells to both \\ activate and \\ respond to \\ vitamin D}

The importance of maintaining a balance between inflammatory responses necessary for pathogen clearance and their timely resolution to prevent tissue damage is clearly exemplified by patients with severe COVID-19, who develop life-threatening hyper-inflammation characterized by high levels of complement activation. Complement itself has been shown to drive both the differentiation of interferon- $\gamma$ $(\mathrm{IFN} \gamma)$-producing $\mathrm{T}$ helper $1\left(\mathrm{~T}_{\mathrm{H}} 1\right)$ cells as well as the eventual shutdown of their pro-inflammatory features through IL-10 expression. This study by Chauss et al. links the complement-mediated retraction of $\mathrm{T}_{\mathrm{H}} 1$ cell responses to vitamin $\mathrm{D}$ receptor (VDR) signalling, which provides a possible mechanistic explanation for the epidemiological associations between vitamin $\mathrm{D}$ deficiency and adverse outcomes in COVID-19 and potentially other infectious diseases.

Single-cell RNA sequencing of $\mathrm{CD}^{+} \mathrm{T}$ cells from the blood and bronchoalveolar lavage fluid (BALF) of eight patients with COVID-19 showed that they have a prominent pro-inflammatory $\mathrm{T}_{\mathrm{H}} 1$ cell signature, enriched for IFN $\gamma$

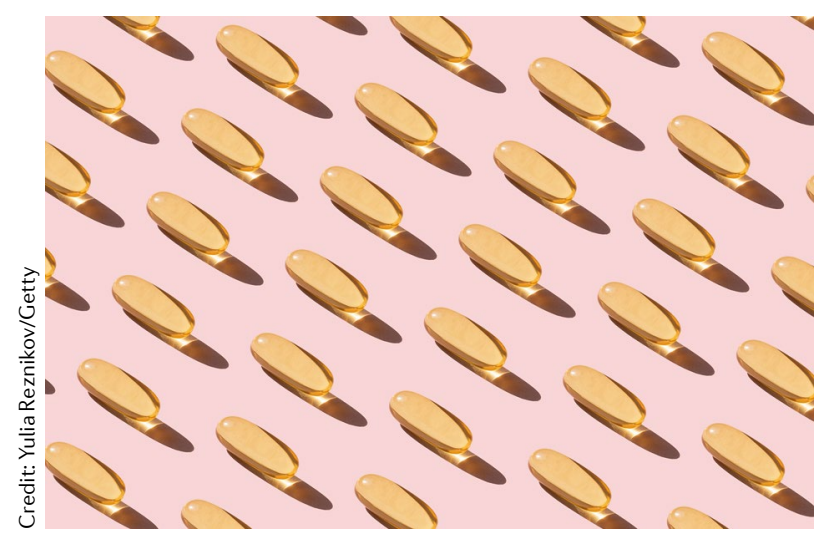

and complement pathway genes, but with lower levels of expression of IL10 than $\mathrm{CD}^{+} \mathrm{T}$ cells from healthy controls. To better understand the pro-inflammatory nature of these COVID-19-associated $\mathrm{T}_{\mathrm{H}} 1$ cells, the authors looked in more detail at the previously described effects of complement on contraction of $\mathrm{T}_{\mathrm{H}} 1$ cell responses. Transcriptomic analysis of $\mathrm{CD} 4^{+} \mathrm{T}$ cells activated with anti-CD3 and anti-CD46 (the receptor for complement component $\mathrm{C} 3 \mathrm{~b}$ ) showed upregulation of both VDR and CYP27B1, which encodes the enzyme catalysing the final activation step of vitamin D. This suggests that complement stimulates T cells to both activate and respond to vitamin $\mathrm{D}$. T cells from CD46-deficient individuals or T cells treated with an inhibitor that blocks intracellular generation of $\mathrm{C} 3 \mathrm{~b}$ did not upregulate VDR or CYP27B1. In response to inactive vitamin $D$, $\mathrm{T}$ cells stimulated with anti-CD3 and anti-CD46 repressed IFN $\gamma$ expression and upregulated IL-10 expression, suggesting that complement-induced VDR signalling promotes shutdown of $\mathrm{T}_{\mathrm{H}} 1$ cell responses.

Further transcriptomic and proteomic analysis showed that vitamin $\mathrm{D}$ promotes the production of IL- 10 by inducing expression of IL-6, IL-6 receptor (IL-6R) and the transcription factor STAT3, leading to STAT3 phosphorylation and activation downstream of IL-6R. Vitamin D also induced genome-wide changes in histone acetylation and recruitment of transcription factors, including at STAT3, IL1O and BACH2 loci. A significant proportion of vitamin $\mathrm{D}$-driven transcription was shown to be $\mathrm{BACH} 2$ dependent, including $I L 6 R$ transcription. Indeed, vitamin $\mathrm{D}$-treated $\mathrm{CD} 4^{+} \mathrm{T}$ cells from a $B A C H 2$ haploinsufficient individual did not upregulate the expected vitamin D-dependent gene set, and the IL-6-STAT3-IL-10 signalling axis was disrupted.

Given the observed association of COVID-19 severity with vitamin D deficiency, the authors looked at the expression of vitamin D-regulated genes in the BALF CD4 ${ }^{+} \mathrm{T}$ cells of patients with COVID-19. On a percell basis, they observed a reciprocal relationship between the expression of $\mathrm{T}_{\mathrm{H}} 1$ cell-associated genes and vitamin D-modulated genes. The results suggest that in patients with severe COVID-19, who have a strongly pro-inflammatory $\mathrm{T}_{\mathrm{H}} 1$ cell phenotype, the transcriptional response to vitamin D is impaired, leading to a failure to switch to IL-10 production and inflammatory resolution. This could be a result of vitamin D insufficiency or some other dysregulation of complement-induced autoregulatory VDR signalling; in either case, clinical trials may help shed light on whether there is clinical benefit to using vitamin $\mathrm{D}$ as an adjunct therapy for COVID-19. Furthermore, IL-6 is generally thought to be a pro-inflammatory cytokine involved in cytokine storm responses, but the results also suggest that vitamin $\mathrm{D}$ as adjunct therapy for COVID-19 might divert IL-6 to pro-resolution functions and thus provide an alternative to blocking IL-6R signalling. The researchers did not test vitamin $\mathrm{D}$ as an adjunct treatment for COVID-19 and caution the results should not be taken as a clinical recommendation.

Kirsty Minton

ORIGINAL ARTICLE Chauss, D. et al. Autocrine vitamin $D$ signaling switches off pro-inflammatory programs of $\mathrm{T}_{\mathrm{H}} 1$ cells. Nat. Immunol. https://doi. org/10.1038/s41590-021-01080-3 (2021) 